\title{
Imagistic and histopathological description of a cystic nephroma during early second trimester of gestation. Case report
}

\author{
Adelina Staicu1, Roxana Popa-Stanilă², Dan Gheban³ ${ }^{3}$ Liviu Chiriacc,5, Flaviu RV Turcu ${ }^{4,5}$, \\ Gabriela Caracostea ${ }^{1}$, Florin Stamatian ${ }^{2}$
}

1"Iuliu Hatieganu“" University of Medicine and Pharmacy, $1{ }^{\text {st }}$ Dept. of Obstetrics and Gynaecology, ${ }^{2}$ IMOGEN, Centre of Advanced Research Studies, 3"Iuliu Hatieganu“" University of Medicine and Pharmacy, Department of Pathology, ${ }^{4}$ Faculty of Physics, "Babes Bolyai“" University, ${ }^{5}$ National Magnetic Resonance Centre, "Babes Bolyai“" University, Cluj-Napoca, Romania

\begin{abstract}
Cystic nephroma is a rare and benign renal tumour of unknown origin, usually diagnosed in the first years of childhood or during adult life. To our knowledge, there are no records in the literature of this particular tumour being descried prenatally.

We present a case of a fetus diagnosed with cystic nephroma on 16 weeks of gestation. The renal tumour was evaluated by prenatal ultrasound, post mortem with 7T magnetic resonance imaging, and conventional autopsy.
\end{abstract}

Keywords: cystic nephroma, ultrasonography, MRI, prenatal

\section{Introduction}

Renal tumours are unusual findings on prenatal ultrasound, representing only $5-7 \%$ of all congenital tumours. Although they are mostly benign, the differential diagnosis is quite difficult, this fact being explained mainly by the similar imagistic presentation as a large multilocular abdominal mass [1]. In case of a congenital cystic renal mass, first diagnosis considerations include multicystic dysplastic kidney, cystic Wilms tumour, cystic partially differentiated nephroblastoma, cystic clear-cell carcinoma, or cystic renal cell carcinoma [2].

Cystic nephroma (or multilocular cystic nephroma) is a benign renal tumour of unknown pathogenesis, probably having the origin in the metanephric blastema and is being described in the first year of life [1,3].

Received 28.07.2016 Accepted 16.09.2016

Med Ultrason

2017, Vol. 19, No 3, 327-329

Corresponding author: Gabriela Caracostea

"Iuliu Hațieganu“"University of Medicine and

Pharmacy, 1st Dept. of Obstetrics and

Gynaecology,

3 - 5 Clinicilor Street,

400006, Cluj-Napoca, Romania

Phone/Fax: 1 - 40-264450115

E-mail: caracostea1@yahoo.com
We present the case of a fetus diagnosed with cystic nephroma on 16 weeks of gestation. The renal tumour is evaluated by prenatal ultrasound, post mortem-MRI (pmMRI) using a magnetic resonance imaging machine of 7 $\mathrm{T}$ magnetic field and conventional autopsy.

\section{Case report}

A 23-year-old healthy primigravida, Caucasian woman, was referred to our Prenatal Diagnosis Centre on 16 weeks and 4 days of gestation, due to a fetal abdominal mass identified during routine ultrasonography. The mother's personal history did not reveal any exposures to teratogenic drugs or infections during pregnancy. Also, her family history and blood tests were non remarkable. The chromosomal analysis of the amniotic fluid identified a female fetus with normal karyotype.

The ultrasound examination was performed using a Voluson E8-Expert ultrasound machine. The fetal biometry corresponded to 16 weeks and 6 days of gestation. Early morphology identified the fetal heart, the central nervous system, the liver, and the right kidney, having normal ultrasound appearances. The left kidney and stomach could not be seen clearly due to a well-encapsulated, multicystic, hypoechogenic mass, measuring 21/10 $\mathrm{mm}$ in size, having the origin in the left upper quadrant 


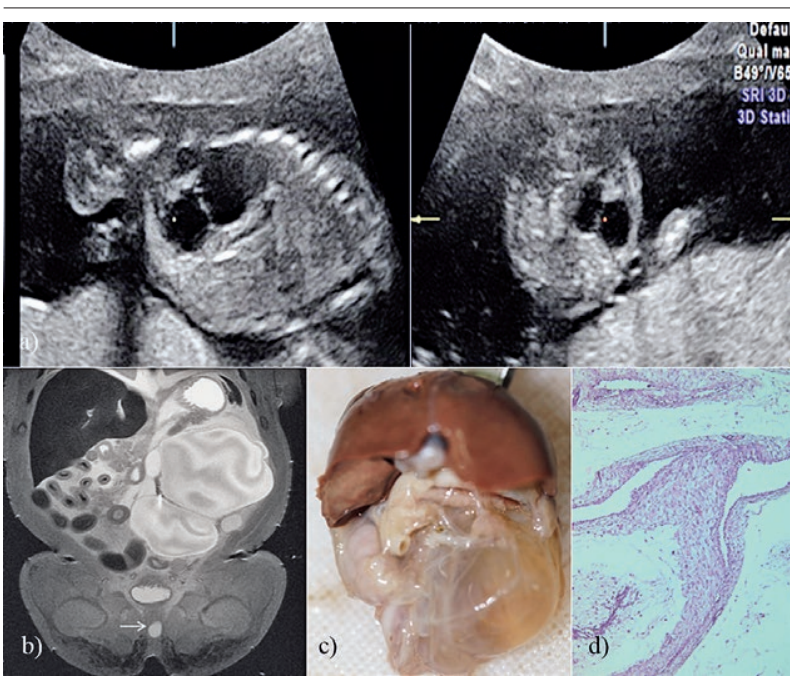

Fig 1. a) Ultrasound examination depicting the fetal abdomen occupied by a well-encapsulated, multicystic, hypoechogenic mass having the origin in the left upper quadrant; b) $7 \mathrm{~T} \mathrm{pm-}$ MRI, T2WI, frontal view of the abdomen describing a large left kidney with a multilocular atypical, fluid-like cysts mass, displacing the abdominal organs; c) Macroscopic aspect of the renal mass from the conventional autopsy; d) HE 10x left kidney presenting cysts lined with flat-to-columnar epithelial cells occasionally producing a "hobnail" appearance.

of the fetal abdomen (fig 1a). Also, excess amniotic fluid was noted.

Due to the gestational age of the pregnancy, the in vivo MRI investigation was postponed until after 18 weeks of gestation.

However, one week after the initial visit, the patient returned with watery discharge per vagina, and was diagnosed with premature rupture of the membranes. Therefore, after informing the parents upon the fetal prognostic, with the mother consent, therapeutic interruption of the pregnancy was performed. The patient delivered a female fetus, weighting $150 \mathrm{~g}$, with a crown to heel length of $19 \mathrm{~cm}$, with no viability signs, a slightly enlarged abdomen, and without any other macroscopic anomalies.

Post-mortem, we performed an MRI-based morphological investigation of the fetus, using a 7 Tesla Bruker Biospec machine. The study protocol was approved by the local Ethics Committee and written informed consent was obtained before the procedures. The fetus was scanned using a Turbo Rare-High Resolution T2 weighted based protocol, after a 24-hour setting in formaldehyde solution. The pm-MRI examination revealed a large, solitary, non-communicating, multilocular cystic mass containing clear fluid, measuring $24 / 19 \mathrm{~mm}$, displacing the abdominal organs in the left upper quadrant. The left suprarenal gland was identified on the superior pole of the mass, strongly suggesting renal origin of the tumour. No haemorrhagic or necrotic components were identified within the specified structure (fig 1b). In addition, left hydroureter and hydrocolpos, secondary to ectopic opening of the ureter in the vagina and partial corpus callosum agenesis were found.

The fetal autopsy report described the left kidney transformed into a mass of $15 / 10 \mathrm{~mm}$, after setting in formaldehyde solution. Histologic examination of the mass, revealed a well-defined tumour with multiple cystic spaces separated by fibrous tissue septae. The cysts were lined with flat-to-columnar epithelial cells occasionally producing a "hobnail" appearance due to the bulging of the nucleus toward the interior of the cyst. There were no solid tissues identified within the tumour, neither mature nephrons nor malignant blastemal cells within the septae of the cystic lesion. The lesion was identified as a cystic nephroma (fig 1c, d).

\section{Discussions}

Cystic nephroma was first described by Edmunds W in 1892 as a "cyst adenoma" [4]. It is a benign tumour, usually encountered in the first years of childhood, affecting mostly males and less common during adult life, when is frequently described in women [5]. Nowadays, cystic nephroma is described together with cystic partially differentiated nephroblastoma (CPDN) as a multilocular cystic renal tumour. Compared to cystic nephroma, CPDN has a low malignant potential and a tendency of recurrence after surgical treatment [6].

The histological characteristic of the renal tumour discovered in our case complies with diagnosis criteria of cystic nephroma established by Powell et al in 1951 [7].

To specifically distinguish cystic nephroma from a CPDN, Joshi et al stated that the cysts tissue of the cystic nephroma must be entirely differentiated and blastematous or embryonal elements must be absent [8], histological characteristic accomplished in all findings of our specimen. Sometimes the Wilms tumour may presents as a multilocular cystic mass, but the lack of haemorrhage, necrosis, or a nodular structure within the cystic mass rules out this possibility in our case [9]. Exceptional, segmental multicystic dysplastic kidney with cysts isolated to a portion of the kidney may have a similar appearance to cystic nephroma, but in this particular case the cysts are not separated by renal parenchyma [5].

To our knowledge, both CPDN and cystic nephroma have not been yet descried prenatally or in the newborn period [6].

Prenatal ultrasound remains the preferred diagnostic method, to identify congenital tumours. However, in the case of multilocular cystic lesions, more detailed imagis- 
tic analysis is needed in order to provide accurate information about the origin and the possibility of invasion to adjacent organs, in order to reduce the differential diagnosis spectrum.

The post mortem examination using 7T MRI revealed reliable information about the origin and the macroscopic characteristic of this particular renal tumour, but the imagistic approach was not sufficient to establish the final diagnosis. Still, the ultrasound and pm-MRI examination obtained important details about the imagistic aspect of these rare renal entities during early second trimester and may provide a guide for future pregnancy management.

Children presenting a cystic renal tumour have a good oncologic outcome after nephrectomy, but the perinatal period is burdened by numerous complications due to the occurrence, in most cases of polyhydramnios [10]. Physiopathology of polyhydramnios in renal tumours is still unfamiliar. In our case could be explained by excessive fetal urine production and ectopic opening of the left ureter in the vagina associated with impaired gastrointestinal reabsorption due to bowel compression.

In conclusion, we described the stages of solving a difficult diagnosis of a rather rare cystic renal tumour at an early gestational age. In order to facilitate the proper patient counselling and treatment strategies, sonographers must be aware that cystic nephroma can be a finding on the prenatal ultrasound especially when a unilateral, cystic renal tumour associates excess amniotic fluid.

\section{References}

1. Alamo E, Beck-Popovic M, Gudinchet F, Meuli R. Congenital tumors: imaging when life just begins. Insights Imaging 2011;2:297-308.

2. Gray GG, Amodio JB, Wood BP. Radiological case of the month. Multilocular cystic Wilms tumor. Arch Pediatr Adolesc Med 1998;152:705-706.

3. Turbiner J, Amin MB, Humphrey PA, et al. Cystic nephroma and mixed epithelial and stromal tumor of kidney: a detailed clinicopathologic analysis of 34 cases and proposal for renal epithelial and stromal tumor (REST) as a unifying term. Am J Surg Pathol 2007;31:489-500.

4. Edmunds W. Cystic adenoma of the kidney. Trans Pathol Soc London 1892;43:89-90.

5. Granja MF, O'Brien AT, Trujillo S, Mancera J, Aguirre DA. Multilocular Cystic Nephroma: A Systematic Literature Review of the Radiologic and Clinical Findings. AJR Am J Roentgenol. 2015;205:1188-1193.

6. Puvaneswary M, Macintosh J, Cassey J. Cystic partially differentiated nephroblastoma. Australas Radiol 2006;50:255-257.

7. Powell T, Shackman R, Johnson HD. Multilocular cysts of the kidney. Br J Urol 1951;23:142-152.

8. Joshi VV, Beckwith JB. Multilocular cyst of the kidney (cystic nephroma) and cystic, partially differentiated nephroblastoma. Terminology and criteria for diagnosis. Cancer 1989;64:466-479.

9. Kurian JJ, Ninan PJ. A rare case of bilateral cystic partially differentiated nephroblastoma recurring as bilateral cystic Wilms tumour. BMJ Case Rep 2015 Apr 15. doi: 10.1136/ bcr-2015-209771.

10. Leclair MD, El-Ghoneimi A, Audry G, et al. The outcome of prenatally diagnosed renal tumors. J Urol 2005;173:186-189. 